\title{
ON CLASSES OF SOLUTIONS OF ELLIPTIC LINEAR PARTIAL DIFFERENTIAL EQUATIONS
}

\section{AVNER FRIEDMAN}

Introduction. Among the theorems which deal with the functional properties of the solutions of elliptic linear partial differential equations, the most important ones are perhaps the following:

(a) The solutions of equations with analytic coefficients are analytic.

(b) The solutions of equations with indefinitely differentiable (i.d.) coefficients are i.d.

In Part I of this paper we prove a result which connects the above mentioned theorems. Qualitatively we prove that the i.d. solutions of elliptic differential systems with i.d. coefficients have the same "distance" from analyticity as have the coefficients. More precisely, we define classes of i.d. functions and show, under certain assumptions, that if the coefficients belong to a certain class, then so do the solutions. In particular, we give a new proof to Theorem (a).

In Part II we define another kind of classes of i.d. functions and prove, for some kinds of elliptic equations, results similar to that of Part I.

All functions in this paper are real functions.

I should like to express my gratitude to Dr. S. Agmon for his help and encouragement during the preparation of Part I of this paper.

\section{PART I}

Let $D$ be a bounded domain in the $N$ dimensional space $E^{N}$ of $N$ real variables $x=\left(x_{1}, \cdots, x_{N}\right)$ and let $\left\{M_{n}\right\}$ be a sequence of positive numbers. We denote by $C\left\{M_{n} ; D\right\}$ the class of all i.d. functions $f(x)$ defined in $D$ and having the following property:

To every closed subdomain $D_{0} \subset D$ there correspond constants $H_{0}$, $H$ such that for $x \in D_{0}$

$$
\left|\frac{\partial^{n} f(x)}{\partial x_{1^{1}}^{i} \cdots \partial x_{N}^{i}}\right| \leqq H_{0} H^{n} M_{n} \quad(n=1,2 \cdots) .
$$

Note that $C\{n ! ; D\}$ is the class of all functions analytic in $D$.

THEOREм 1. Consider the elliptic system of linear partial differential equations

Received by the editors July 19, 1956. 


$$
\sum_{l=1}^{p} A_{k l} u_{l}(x)=h_{k}(x) \quad(k=1, \cdots, p)
$$

where

$$
A_{k l}=\sum_{j=0}^{8} \sum_{j_{1}+\cdots+j_{N}=j} f^{k l}{ }_{j_{1}} \cdots j_{N}(x) \frac{\partial^{j}}{\partial x_{1}^{j_{1}} \cdots \partial x_{N}^{j_{N}}} .
$$

If all the coefficients $f_{j_{1} \ldots j_{N}}^{k l}(x), h_{k}(x)$ belong to $C\left\{M_{n} ; D\right\}$, and if the $M_{n}$ satisfy the following monotonicity conditions:

(3) $\left(\begin{array}{c}n-s+2 \\ i\end{array}\right) M_{i} M_{n+2-i} \leqq A n M_{n+1} \quad(i=2,3, \cdots, n-s+2)$,

where $A$ is some constant, then the components of every solution $\left(u_{1}(x), \cdots, u_{p}(x)\right)$ belong to $C\left\{M_{n} ; D\right\}$.

Note that (3) implies

$$
n M_{n} \leqq A_{1} M_{n+1},
$$

$$
\left(\begin{array}{c}
n-s+1 \\
i
\end{array}\right) M_{i} M_{n+1-i} \leqq A_{2} M_{n+1} \quad(i=1, \cdots, n-s+1)
$$

where $A_{i}$ are some constants.

1.1. The proof will be given for the case $N=3, p=1$, the proof of the general case is analogous. Equation (1) becomes

$$
A u \equiv \sum_{j=0}^{s} \sum_{j_{1}+j_{2}+j_{3}=j} f_{j_{1} j_{2} j_{3}}(x) \frac{\partial^{j} u(x)}{\partial x_{1}^{j_{1} \partial} x_{2}^{j_{2} \partial} x_{z^{3}}^{j_{3}}}=h(x) .
$$

In what follows we shall use the following results due to Lopatinski $[2 ; 3]$ :

Let $D_{0}$ be a closed subdomain of $D$. Then there exists a fundamental solution $\phi(x, y)$ of the equation $A u=0$, defined for $x, y \in D_{0}$ and having the following properties:

(a) $A \phi(x, y)=0$ for every $y \in D_{0}$.

(b) For every function $v(x)$ having $s$ continuous derivatives in $D_{0}$, and for every cube $G^{\prime} \subset D_{0}$ and $y \in G^{\prime}$,

(5) $v(y)=\sum_{j=1}^{3} \int_{S^{\prime}} \cos \left(\nu, x_{j}\right) B^{j}(\phi(x, y), v(x)) d S_{x}+\int_{G^{\prime}} \phi(x, y) A^{*} v(x) d G_{x}$, where $S^{\prime}$ is the boundary of $G^{\prime}, \nu$ is the outwardly directed normal to $S^{\prime}$, 


$$
A^{*} v=\sum_{j=0}^{s} \sum_{j_{1}+j_{2}+j_{3}=j}(-1)^{j} \frac{\partial^{j}}{\partial x_{1}^{j_{1} \partial \partial x_{2}^{j} \partial x_{3}^{j_{3}}}}\left(f_{j_{1} j_{2} j_{3}}(x) v(x)\right)
$$

and the $B^{j}(v, w)$ are defined by

$$
v A^{*} w-w A v=\sum_{j=1}^{3} \frac{\partial}{\partial x_{j}} B^{i}(v, w) .
$$

(c) $\phi(x, y)$ has continuous derivates of all orders for $x \neq y$, and the functions

$$
\begin{aligned}
\psi(x, y)=|x-y|^{8-s+\Sigma j_{i}+\Sigma k_{i}} \frac{\partial^{i+k}}{\partial x_{1}^{j_{1}} \cdots \partial y_{3}^{k_{3}}} \phi(x, y) \\
\left(3-s+\sum j_{i}+\sum k_{i}>0\right),
\end{aligned}
$$

where $|x|$ denotes the norm $\left(\sum x_{i}^{2}\right)^{1 / 2}$, are bounded for $x, y \in D_{0}$, $x \neq y$.

1.2. Let $G$ be a closed cube contained in $D$, with edges parallel to the coordinate axes, and of a diameter to be determined later. Denote by $v^{(n)}(x)$ any $n$th (partial) derivative of $v(x)$, and by $|v|_{\delta}$ the maximum of $|v(x)|$ in a cube homothetic with $G$ and such that the distance between its surface and the surface $S$ of $G$ is $\delta$. We shall prove by induction that

$$
\left|u^{(k)}\right|_{\delta} \leqq \frac{H_{0} H^{k}}{\delta^{k}} M_{k}
$$

for all the partial derivatives $u^{(k)}(x)$. The proof of Theorem 1 is then completed by using the Heine-Borel Theorem.

We may assume (8) to hold for $k \leqq s$, since we can take $H_{0}$ large enough. Assume (8) to hold for $k \leqq n(n \geqq s)$ and differentiate (4) $n-s+2$ times. We obtain

$$
\begin{aligned}
& \sum_{j_{1}+j_{2}+j_{3}=s} f_{j_{1} j_{2} j_{3}} \frac{\partial^{s} u^{(n-s+2)}}{\partial x_{1}^{j_{1} \partial} x_{2}^{j_{2} \partial} x_{3}^{j_{3}}} \\
& \text { (9) }=-\sum_{i=1}^{n-s+2} \sum_{j_{1}+j_{2}+j_{3}=s}\left(\begin{array}{c}
n-s+2 \\
i
\end{array}\right) f_{j_{1} j_{2} j_{3}}^{(i)} \frac{\partial^{s} u^{(n-s+2-i)}}{\partial x_{1}^{j_{1}} \partial x_{2}^{j_{2} \partial} \partial x_{3}^{j_{3}}} \\
& -\sum_{i=0}^{n-s+2} \sum_{j=0}^{s-1} \sum_{j_{1}+j_{2}+j_{s}=j}\left(\begin{array}{c}
n-s+2 \\
i
\end{array}\right) f_{j_{1} j_{2} j_{3}}^{(i)} \frac{\partial^{i} u^{(n-s+2-i)}}{\partial x_{1}^{j} \partial x_{2}^{j} \partial x_{3}^{j_{3}}}+h^{(n-s+2)} \\
& \equiv G(x) \text {. }
\end{aligned}
$$

Denoting

$$
\bar{A} v(x)=\sum_{j_{1}+j_{2}+j_{3}=8} f_{j_{1} j_{2} j_{3}}(x) \frac{\partial^{s} v(x)}{\partial x_{1}^{j} \partial x_{2}^{j_{2} \partial} x_{3^{j}}^{j_{3}}},
$$


we can write (9) in the form

$$
\bar{A} u^{(n-8+2)}(x)=G(x) .
$$

Let $y \in G$ be a point whose distance from $S$ is $\delta^{\prime}$, and let

$$
\delta=\delta^{\prime}\left(1-\frac{1}{n}\right)
$$

Denote by $G_{\delta}$ a cube with center $y$, and edges parallel to the coordinate axes of length $2\left(\delta^{\prime}-\delta\right)$. Let $S_{\delta}$ be the surface of $G_{\delta}$. Applying the results stated in 1.1 to the elliptic operator $\bar{A}^{*}$, with $v(x)$ $=u^{(n-s+2)}(x), G_{\delta}$ instead of $G^{\prime}$ and $D_{0} \supset G$, we obtain

$$
\begin{aligned}
u^{(n-s+2)}(y)= & \int_{S_{\delta}} \sum_{j=1}^{z} \cos \left(\nu, x_{j}\right) \bar{B}^{j}\left(\phi(x, y) u^{(n-s+2)}(x)\right) d S_{x} \\
& +\int_{G_{\delta}} \phi(x, y) \bar{A} u^{(n-s+2)}(x) d G_{x}=\bar{J}_{1}+\bar{J}_{2},
\end{aligned}
$$

so that

$$
u^{(n+1)}(y)=\bar{J}_{1}^{(8-1)}+\bar{J}_{2}^{(\iota-1)}=J_{1}+J_{2} .
$$

We now evaluate $J_{2}$ and $J_{1}$. In the sequel $B_{1}, \cdots, B_{15}$ will be used to denote appropriate constants, independent of $n$. Using the boundedness of the $\psi(x, y)$ in (7), we get

$$
\left|J_{2}\right| \leqq B_{1}\left|\bar{A} u^{(n-\delta+2)}\right|_{\delta} \int_{G_{\delta}} \frac{d G_{x}}{|x-y|^{2}} \leqq \frac{B_{2} \delta^{\prime}}{n}|G|_{\delta} .
$$

From the definition of $G(x)$ in (9), the inductive assumption, (3) and (12), we obtain, by taking $H$ to be sufficiently large (but independent of $n$ )

$$
|G|_{\delta} \leqq B_{3} n\left|u^{(n+1)}\right|_{\delta}^{*}+B_{4} \frac{H_{0} H^{n}}{\delta^{\prime n+1}} n M_{n+1},
$$

where $\left|u^{(n+1)}\right|_{\delta}^{*}$ denotes

$$
\max _{i_{1}+i_{2}+t_{2}=n+1}\left|\frac{\partial^{n+1} u}{\partial x_{1}^{i} \partial x_{2^{2} \partial x_{3}^{i}}^{i}}\right| \delta .
$$

From (15), (16),

$$
\left|J_{2}\right| \leqq B_{5} R\left|u^{(n+1)}\right|_{\delta}^{*}+\frac{B_{6} H_{0} H^{n}}{\delta^{\prime n+1}} M_{n+1},
$$


where $R$ is the diameter of $G$.

To evaluate $J_{1}$, observe that it is sufficient to evaluate $I^{(s-1)}$, where $I$ is of the form

$I=\int_{S_{\delta}}\left(u^{(n-s+2)}(x) f(x)\right)^{(i)}(\phi(x, y))^{(s-i-1)} \cos \left(\nu x_{j}\right) d S_{x}(0 \leqq i \leqq s-1)$.

Here $f(x)$ denotes any of the functions $f_{j_{1} j_{2} j_{3}}(x), \sum j_{i}=s$. For $0 \leqq i$ $<s-1$ we easily obtain, by using $\left(3^{\prime}\right),(12)$, the inductive assumption and the boundedness of the $\psi(x, y)$ in $(7)$,

$$
\left|I^{(8-1)}\right| \leqq \frac{B_{7} H_{0} H^{n}}{\delta^{\prime n+1}} M_{n+1} .
$$

Take now $i=s-1$, then

$$
\begin{aligned}
& \left(u^{(n-s+2)}(x) f(x)\right)^{(s-1)} \\
& \quad=u^{(n+1)}(x) f(x)+\sum_{j=0}^{s-2}\left(\begin{array}{c}
s-1 \\
j
\end{array}\right) f^{(s-1-j)}(x) u^{(n-s+2-j)}(x) .
\end{aligned}
$$

Each term corresponding to

$$
f^{(s-1-j)}(x) u^{(n-s+2-j)}(x) \quad(0 \leqq j \leqq s-2)
$$

is evaluated in the same way we have evaluated $I^{(s-1)}$ in the previous case. There remains the term corresponding to $u^{(n+1)}(x) f(x)$.

To evaluate the $(s-1)$ th derivative of

$$
I_{0}=\int_{s_{\delta}} \phi(x, y) f(x) u^{(n+1)}(x) d S_{x}
$$

let us make the restrictive assumption that $u^{(n+1)}(x)$ can be written in each one of the following forms:

$$
\frac{\partial}{\partial x_{i}} u^{(n)}(x) ; \quad \frac{\partial}{\partial x_{j}} u^{(n)}(x)
$$

If we evaluate $I_{0}$ by means of integration by parts and use $\left(3^{\prime}\right),(12)$, the inductive assumption and the boundedness of the $\psi(x, y)$, we obtain

$$
\left|I_{0}^{(8-1)}\right| \leqq \frac{B_{8} H_{0} H^{n}}{\delta^{\prime n+1}} M_{n+1} .
$$

From (14), (17), (18) and (20) it follows that

$$
\left|u^{(n+1)}(y)\right| \leqq B_{5} R\left|u^{(n+1)}\right|_{\delta}^{*}+\frac{B_{9} H_{0} H^{n}}{\delta^{\prime n+1}} M_{n+1} .
$$


Since $y$ is an arbitrary point whose distance from $S$ is $\delta^{\prime}$, we get

$$
\left|u^{(n+1)}\right|_{\delta^{\prime}} \leqq B_{5} R\left|u^{(n+1)}\right|_{\delta}^{*}+\frac{B_{9} H_{0} H^{n}}{\delta^{\prime n+1}} M_{n+1} \text {. }
$$

Because of the restriction made in evaluating $I_{0}^{(8-1)}$, the inequality (22) does not cover only the cases of $\partial^{n+1} u(x) / \partial x_{i}^{n+1}$. For suppose, for instance, we have to evaluate $\partial^{n+1} u(x) / \partial x_{1}^{i} \partial x_{2}^{j} \partial x_{3}^{k}, i>0, j>0$. Since $n-s+2 \geqq 2$, we may assume that already in (11)

$$
u^{(n-s+2)}(x)=\frac{\partial}{\partial x_{1}} u^{(n-s+1)}(x)=\frac{\partial}{\partial x_{2}} u^{(n-s+1)}(x)
$$

and we can integrate by parts in $I_{0}^{s-1}$. We shall evaluate for example, $\partial^{n+1} u(x) / \partial x_{1}^{n+1}$. Write (4) in the form

$$
\begin{aligned}
f_{s 00}(x) \frac{\partial^{s} u}{\partial x_{1}^{s}}= & -\sum_{j_{1}+j_{2}+j_{3}-s}^{\prime} f_{j_{1} j_{2} j_{3}} \frac{\partial^{s} u}{\partial x_{1}^{j_{1}} \partial x_{2}^{j_{2}} \partial x_{3}^{j_{3}}} \\
& -\sum_{j=0}^{-1} \sum_{j_{1}+j_{2}+j_{3}-j} f_{j_{1} j_{2} j_{3}} \frac{\partial^{j} u}{\partial x_{1}^{j_{1}} \partial x_{2}^{j_{2} z} \partial x_{3}^{j_{s}}}+h
\end{aligned}
$$

and differentiate it $n-s+1$ times with respect to $x_{1}$. Using ( $\left.3^{\prime \prime}\right),(22)$ and the inductive assumption, we get

$$
\left|f_{800} \frac{\partial^{n+1} u}{\partial x_{1}^{n+1}}\right|_{\delta^{\prime}} \leqq B_{11} R\left|u^{(n+1)}\right|_{\delta}^{*}+\frac{B_{10} H_{0} H^{n}}{\delta^{\prime n+1}} M_{n+1} .
$$

Since equation (4) is elliptic, $f_{800}(x) \neq 0$, and so (24) implies

$$
\left|\frac{\partial^{n+1} u}{\partial x_{1}^{n+1}}\right| \zeta^{\prime} \leqq B_{13} R\left|u^{(n+1)}\right|_{\delta}^{*}+\frac{B_{12} H_{0} H^{n}}{\zeta^{\prime n+1}} M_{n+1} \text {. }
$$

From (22), (25) we conclude

$$
\left|u^{(n+1)}\right|_{\delta^{\prime}}^{*} \leqq B_{14} R\left|u^{(n+1)}\right|_{\delta}^{*}+B_{15} \frac{H_{0} H^{n}}{\delta^{\prime n+1}} M_{n+1} .
$$

Taking $R<\left(10 B_{14}\right)^{-1}$ we get

$$
\left|u^{(n+1)}\right|_{\delta^{\prime}}^{*} \leqq \frac{1}{10}\left|u^{(n+1)}\right|_{\delta}^{*}+\frac{B_{15} H_{0} H^{n}}{\delta^{\prime n+1}} M_{n+1} .
$$

We now apply the following

Lemma. Let $g(t)$ be a positive monotone-decreasing function, defined in the interval $0 \leqq t \leqq t_{0}$ and satisfying 


$$
g(t) \leqq \frac{1}{10} g\left(t\left(1-\frac{1}{n}\right)\right)+\frac{C}{t^{n+1}} \quad(C>0),
$$

then $g(t) \leqq 5 C / t^{n+1}$.

The proof of the lemma follows easily, by repeatedly applying (27). Applying the lemma to $g(t)=\left|u^{(n+1)}\right|_{t}^{*}$, we obtain from (26)

$$
\left|u^{(n+1)}\right|_{\delta}^{*} \leqq \frac{5 B_{15} H_{0} H^{n}}{\delta^{n+1}} M_{n+1} \text {. }
$$

Taking $H>5 B_{15}$ we arrive at (8) with $k=n+1$, which completes the proof of the theorem.

1.3. Corollary. Let the $M_{n}$ be chosen so that $C\left\{M_{n} ; D\right\}$ is a quasianalytic class (see [4]). Then $u_{1}, \cdots, u_{p}$ belong to the same class, and therefore they possess the following uniqueness property: If $u_{k}(x)$ vanishes at a point $x^{0} \in D$ together with its derivatives of all orders, then it vanishes identically in $D$.

This property of the $u_{k}(x)$ is trivial when $u_{k}(x)$ is analytic. The problem whether this property holds for general nonanalytic elliptic equations is still open.

1.4. If the coefficients of an elliptic linear equation are not analytic, then the solutions may also be nonanalytic. This is shown by the example of

$$
\Delta u+f(x) u=0, \quad u=1+e^{-1 / x_{1}^{2}} .
$$

Here, both $f(x)$ and $u(x)$ do not belong to any quasi-analytic class.

1.5. Theorem 1 is not true if no monotonicity conditions are assumed on the $\left\{M_{n}\right\}$. This is shown by the example of

$$
\frac{\partial^{2} u}{\partial x_{1}^{2}}+\frac{\partial^{2} u}{\partial x_{2}^{2}}=0, \quad u(x)=\operatorname{Re}\left\{1-\left(x_{1}+i x_{2}\right)\right\}^{-1} .
$$

Here, the coefficients of the equation belong to every subclass of $C\{n ! ; D\}$, but $u(x)$ does not belong to any real subclass of $C\{n ! ; D\}$. In Part II we define another kind of classes of i.d. functions which are especially adopted for cases where the coefficients of the elliptic equations are analytic.

1.6. An analogue of Theorem 1 can be proved for the solutions of certain kinds of parabolic equations. This will appear in a later paper in Duke Mathematical Journal.

\section{PART II}

Let $D$ be a bounded $N$-dimensional domain, and let $\left\{M_{n}\right\}$ be a 
sequence of positive numbers. We denote by $K\left\{M_{n} ; D\right\}$ the class of all i.d. functions $f(x)$ defined in $D$ and having the following property: To every closed subdomain $D_{0} \subset D$ there correspond constants $H_{0}$, $H$ such that for $x \in D_{0}$

$$
\left|\Delta^{n} f(x)\right| \leqq H_{0} H^{n} M_{n} \quad(n=0,1, \cdots) .
$$

For instance (see [1]), the class $K\{(2 n)$ !; $D\}$ is the class of all functions which are analytic in $D$.

In Part I we were interested in classes which contained $C\{n ! ; D\}$. In this part we shall be interested, as already mentioned in 1.5 , in subclasses of $K\{(2 n) ! ; D\}$. We shall prove results of the same character as that of Theorem 1 .

Theorem 2. Let $u(x)$ be a solution in a bounded domain $D$ of the elliptic linear equation

$$
\Delta^{m} u+P_{1}(x) \Delta^{m-1} u+\cdots+P_{m}(x) u=0,
$$

where $P_{i}(x)$ is a polynomial of degree $i$ in $x_{1}, \cdots, x_{N}$. Then

$$
u(x) \in K\{n ! ; D\} .
$$

2.1. The proof will be given in the case of $N=3$ and for the equation

$$
\Delta^{2} u(x)+\stackrel{2}{x_{1}} u(x)=0 .
$$

The proof of the general case, although more complicated, is in the same method.

Take $V \subset D$ to be a sphere of radius $R(R<1)$ and let $S$ be its surface. Denote by $|v|_{\delta}$ the max. of $|v(x)|$ in a concentric sphere of radius $R-\delta$, and by $|\partial v(x) / \partial x|$ the max. of the absolute values of the first partial derivatives of $v(x)$ with respect to all directions, at the point $x$. We shall prove by induction that

$$
\left|\Delta^{k} u\right|_{\delta} \leqq \frac{H_{0} H^{k}}{\delta^{k}} k ! .
$$

Then, the using Heine-Borel Theorem, Theorem 2 follows.

Assume (4) to hold for $k \leqq n+1$. In the sequel, $B_{1}, \cdots, B_{7}$ will be used to denote appropriate constants.

Let $y \in V$. Denote its distance from $S$ by $\delta^{\prime}$ and let

$$
\delta=\delta^{\prime}(1-\alpha / n),
$$

where $\alpha$ is a positive number which satisfies $e^{\alpha}<2$.

We use Green's Identity 
(6) $-4 \pi \Delta^{n-1} u(y)=\int_{V_{\delta}} \frac{\Delta^{n} u}{r} d V-\int_{S_{\delta}}\left(\Delta^{n-1} u \frac{\partial}{\partial \nu} \frac{1}{r}-\frac{\partial \Delta^{n-1} u}{\partial \nu} \frac{1}{r}\right) d S$, where $V_{\delta}$ is a sphere of radius $\delta^{\prime}-\delta$ and center $y$, and $S_{\delta}$ is its surface. Differentiating (6) we get

(7) $\left|\frac{\partial}{\partial y} \Delta^{n-1} u(y)\right| \leqq B_{1}\left(\delta^{\prime}-\delta\right)\left|\Delta^{n} u\right|_{\delta}+\frac{B_{1}\left|\Delta^{n-1} u\right|_{\delta}}{\delta^{\prime}-\delta}+\frac{1}{2}\left|\frac{\partial}{\partial x} \Delta^{n-1} u\right|_{\delta}$.

Using (5) and the inductive assumption, we obtain

$$
\left|\frac{\partial}{\partial \mathrm{x}} \Delta^{n-1} u\right|_{\gamma^{\prime}} \leqq \frac{B_{2} H_{0} H^{n}}{\delta^{\prime n}} n !+\frac{1}{2}\left|\frac{\partial}{\partial \mathrm{x}} \Delta^{n-1} u\right|_{\delta}
$$

It follows from (8), by using an argument similar to that of the lemma in 1.3 , that

$$
\left|\frac{\partial}{\partial x} \Delta^{n-1} u\right|_{\delta} \leqq \frac{B_{3} H_{0} H^{n}}{\delta^{n}} n !
$$

Similarly we derive

$$
\left|\frac{\partial}{\partial \mathrm{x}} \Delta^{n-2} u\right|_{\delta} \leqq \frac{B_{4} H_{0} H^{n-1}}{\delta^{n-1}}(n-1) !
$$

Differentiating twice the identity

$$
\begin{aligned}
-4 \pi \Delta^{n-2} u(y) & =\frac{1}{2 !} \int_{V_{\delta}} r \Delta^{n-1} u d V \\
& -\frac{1}{2 !} \int_{S \delta}\left(r^{2} \Delta^{n-1} u \frac{\partial}{\partial \nu} \frac{1}{r}-r^{2} \frac{\partial \Delta^{n-1} u}{\partial \nu} \frac{1}{r}\right) d S \\
& -\int_{S_{\delta}}\left(\Delta^{n-2} u \frac{\partial}{\partial \nu} \frac{1}{r}-\frac{\partial}{\partial \nu} \Delta^{n-2} u \cdot \frac{1}{r}\right) d S
\end{aligned}
$$

and using (10) and the inductive assumption, we obtain

$$
\begin{aligned}
\left|\frac{\partial^{2}}{\partial y_{i} \partial y_{j}} \Delta^{n-2} u(y)\right| \leqq & B_{5}\left(\delta^{\prime}-\delta\right)^{2}\left|\Delta^{n-1} u\right|_{\delta}+B_{5}\left|\Delta^{n-1} u\right|_{\delta} \\
& +B_{5}\left(\delta^{\prime}-\delta\right)\left|\frac{\partial}{\partial x} \Delta^{n-1} u\right|_{\delta}+\frac{B_{5}\left|\Delta^{n-2} u\right|_{\delta}}{\left(\delta^{\prime}-\delta\right)^{2}} \\
& +\frac{B_{5}}{\delta^{\prime}-\delta}\left|\frac{\partial}{\partial x} \Delta^{n-2} u\right|_{\delta} \leqq \frac{B_{6} H_{0} H^{n}}{\delta^{\prime n}} n !
\end{aligned}
$$

and in particular 


$$
\left|\frac{\partial^{2} \Delta^{n-2} u}{\partial x_{1}^{2}}\right|_{\delta} \leqq \frac{B_{6} H_{0} H^{n}}{\delta^{n}} n !
$$

On using (9), (10), (12), we conclude from the identity

(13) $\Delta^{n}\left(x_{1}^{2} u\right)=n(n-1) \frac{\partial^{2}}{\partial x_{1}^{2}} \Delta^{n-2} u+2 n x_{1} \frac{\partial}{\partial x_{1}} \Delta^{n-1} u+n \Delta^{n-1} u+x_{1}^{2} \Delta^{n} u$, that

$$
\left|\Delta^{n}\left(x_{1}^{2} u\right)\right|_{\delta} \leqq \frac{B_{7} H_{0} H^{n}}{\delta^{n}}(n+2) !
$$

and together with (3) we finally obtain

$$
\left|\Delta^{n+2} u\right|_{\delta} \leqq \frac{B_{7} H_{0} H^{n}}{\delta^{n}}(n+2) ! .
$$

Taking $H \geqq B_{7}^{1 / 2}$, the proof of (4) is completed.

2.2. The examples

$$
\begin{aligned}
& u=e^{x_{1}^{2}} \notin K\{(\vartheta n) ! ; D\}, \vartheta<1, \quad \Delta u-\left(4 x_{1}^{2}+2\right) u=0, \\
& u=e^{x_{1}^{3}} \notin K\{n ! ; D\}, \quad \Delta u-\left(9 x_{1}^{4}+6 x_{1}\right) u=0
\end{aligned}
$$

show that Theorem 2 is not far from being sharp.

2.3. The method used to prove Theorem 2, can be used to prove

Theorem 3. Let $u(x)$ be a solution in a bounded domain $D \subset E^{N}$ of the linear elliptic equation

$$
\Delta^{m} u+\sum_{j=0}^{m-1} \sum_{k=1}^{m-j} \sum_{i_{1}, \cdots, i_{k}=1}^{N} \alpha_{i_{1} \cdots i_{k}}^{j} \frac{\partial^{k}}{\partial x_{i_{1}} \cdots \partial x_{i_{k}}} \Delta^{i} u=0,
$$

where $a_{i_{1}}^{j} \ldots i_{k}$ are constants. Then $u(x) \in K\{n ! ; D\}$.

Obviously, we may combine Theorems 2 and 3 to obtain a more general statement.

\section{REFERENCES}

1. N. Aronszajn, Sur une théorème de la théorie des fonctions analytiques de plusieurs variables complexes, C. R. Acad. Sci. Paris vol. 205 (1937) pp. 16-18.

2. Y. B. Lopatinski, Fundamental systems of solutions of linear differential equations of elliptic type, C.R. (Doklady) Acad. Sci. URSS. vol. 78 (1951) pp. 433-436.

3. - Normal fundamental solutions of systems of linear differential equations of elliptic type, C. R. (Doklady) Acad Sci. URSS. vol. 78 (1951) pp. 865-867.

4. S. Mandelbrojt, Séries de Fourier et classes quasi-analytigues de fonctions, Collection Borel, Paris, 1935.

The Hebrew University, Jerusalem 